Performance and Phase I Upgrade Plans for the CMS Pixel Detector

\author{
Hanno Perrey
}

DESY, Hamburg

On behalf of the CMS collaboration

October 31st,

2012 IEEE Nuclear Science Symposium 


\section{The CMS Pixel Tracker}

- The silicon pixel detector is the innermost component of the

CMS tracking system.

- It consists of 3 layers in the barrel (BPIX, 48M pixels) and $2+2$ disks in the forward region (FPIX, 18M pixels).

- Pixel size: $100 \mu \mathrm{m} \times 150 \mu \mathrm{m}$

- Plays crucial role in full tracking, triggering, primary vertex finding, $e^{-} / \gamma$ identification, $\mu$ reconstruction, $\tau$ identification, $b$-tagging
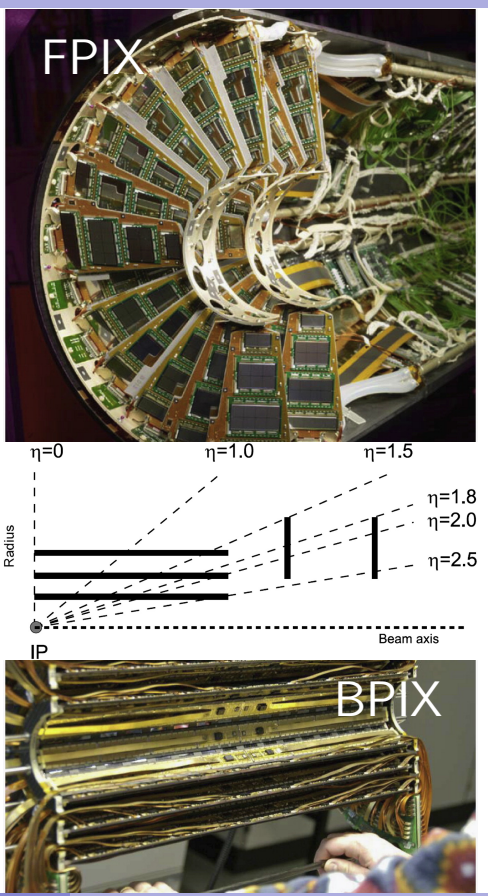


\section{Current Tracker Performance}

- Fully operational ( 96\%)

- Stable running, small contributions to downtime

- Hit efficiency ( $\geq 99 \%)$ and resolution excellent as expected

- Radiation damage clearly visible from leakage currents which

- rise with fluence (luminosity)

- anneal with temperature (shutdowns/operation)

$\Rightarrow$ Layer 1 needs to be replaced in the foreseeable future (as planned)
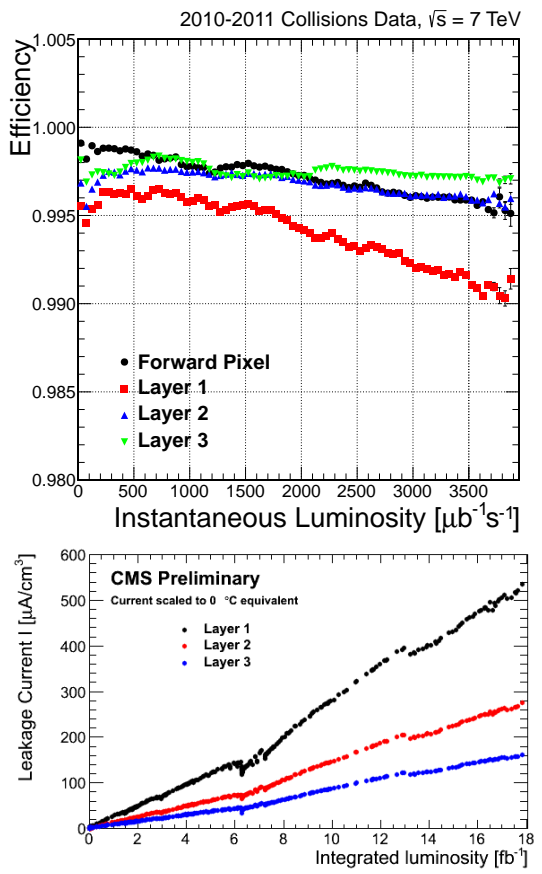


\section{Why upgrade?}

Excellent LHC performance $\rightarrow$ increasingly challenging running conditions!

- Pixel tracker designed for and performing very well at instantaneous luminosity of up to $1 \times 10^{34} \mathrm{~cm}^{-2} \mathrm{~s}^{-1} \Rightarrow$ expected 2014 !

- At $2 \times$ design luminosity (likely reached before 2018) limitations of pixel due to:

- data loss due to limits of readout chip buffers and speed

- tracking inefficiency/higher fake rates due to difficult pattern recognition

- reduced hit resolution \& detection efficiency due to radiation damage

CMS Total Integrated Luminosity, p-p

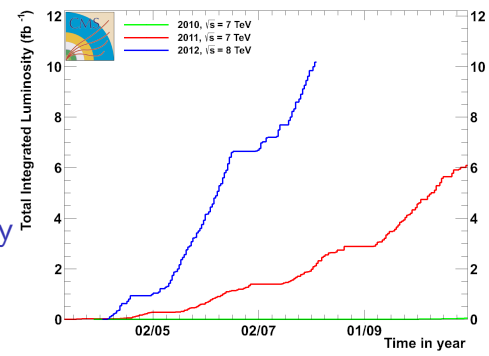

- Goal for upgrade: maintain high efficiency and low fake rates of current pixel detector at 50 interactions per bunch-crossing (pile-up) or higher

- Schedule: planned for year-end technical stop 2016/2017

- Constrains: Need to minimize risks and downtime $\Rightarrow$ use existing services 


\section{Sensor Technology for the Upgrade}

- Keep present design: $285 \mu \mathrm{m}$ thick DOFZ Si pixel size of $100 \times 150 \mu \mathrm{m}^{2}$,

- $\mathrm{n}^{+}$-in-n: (already) radiation hard:

- fluence of $1.5 \times 10^{15} \mathrm{n}_{\mathrm{eq}} / \mathrm{cm}^{2}$ expected during lifetime of Layer 1

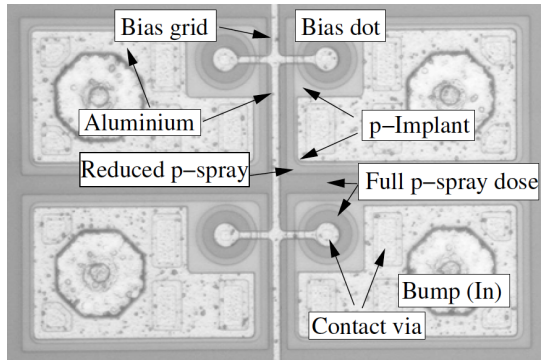

- for samples irradiated to $1.1 \times 10^{15} \mathrm{n}_{\mathrm{eq}} / \mathrm{cm}^{2}$ bias of 450 to $600 \mathrm{~V}$ sufficient

- also gain from improvements on thresholds (see next slide)

$\Rightarrow$ Layer 1 can be efficiently operated

- p-spray (BPIX) or p-stop (FPIX) for n-side isolation

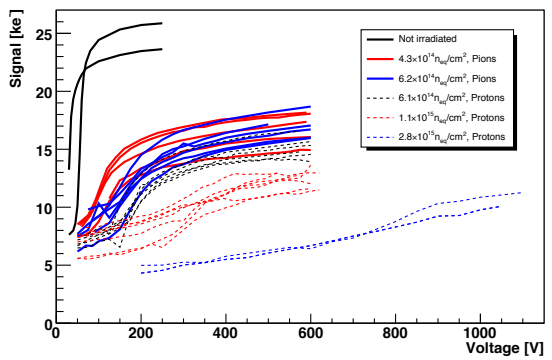




\section{New Readout Chip (ROC) Design}

Current ROC:

- dead time free at low pixel hit rates, but

- up to $4 \%$ data loss at design luminosity

- up to $16 \%$ data loss at $2 \times$ design lumi

Upgraded ROC design:

- increased readout link speed

( $40 \mathrm{MHz}$ analog $\rightarrow 160 \mathrm{MHz}$ digital)

- reduced data loss by additional buffers and increased buffer sizes

- enhanced analog performance:

- lower charge thresholds possible (reduced internal cross-talk)

- reduction of time-walk effects (faster pixel cell comparator)

$\Rightarrow$ improved performance

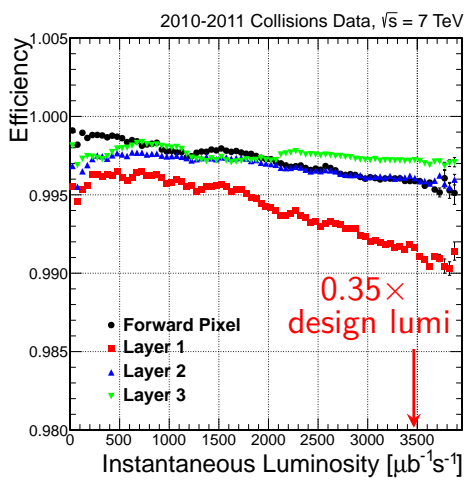
in the presence of radiation damage 


\section{Pixel Detector Geometry: From 3 to 4 track points}

- optimized detector layout for 4-hit coverage over full $\eta$ range with minimal innermost layer radius

$\Rightarrow$ improves efficiency and resolution for pixel-only tracks important for:

- High Level Triggering

- full-track seeding $\Rightarrow$ higher full track efficiency \& lower fake track rate

- vertexing: both primary ( $\rightarrow$ pile-up) and secondary $(\rightarrow b$-tagging)

- keep to available services:

- DC-DC converters for increased power requirements

- digital pixel read-out to meet bandwidth needs
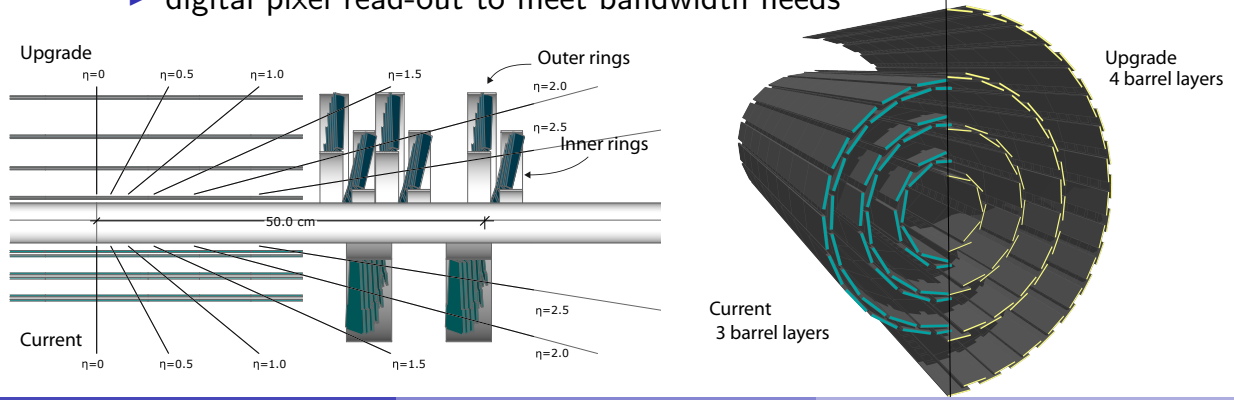


\section{Lower Material Budget}

- even with additional layer ( $\sim 50 \%$ more modules!), reduce material budget through:

- two-phase $\mathrm{CO}_{2}$ cooling (low mass \& small pipes possible)

- ultra-lightweight mechanical support

- moving passive material (electronic boards and connections) out of the tracking volume
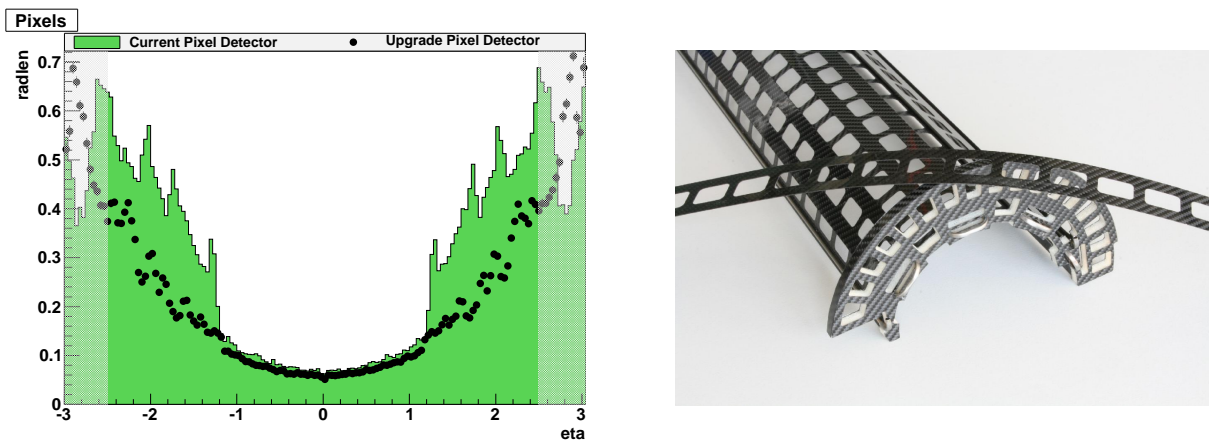


\section{Expected Performance: $b$-Taggin}

- $b$-tagging relies on track impact parameter \& primary vertex resolutions $\rightarrow$ sensitive to improvements in upgraded detector

- study performed on simulated $t \bar{t}$ sample with Combined Secondary Vertex algorithm $\mathrm{w} / \mathrm{o}$ any tuning w.r.t. pile-up (PU), upgraded detector geometry or algorithm settings

- $b$-tagging performance significantly better for upgraded detector:

- at zero PU: result of 4th pixel layer \& smaller inner layer radius

- at PU of 50: still better than current detector at PU of 25
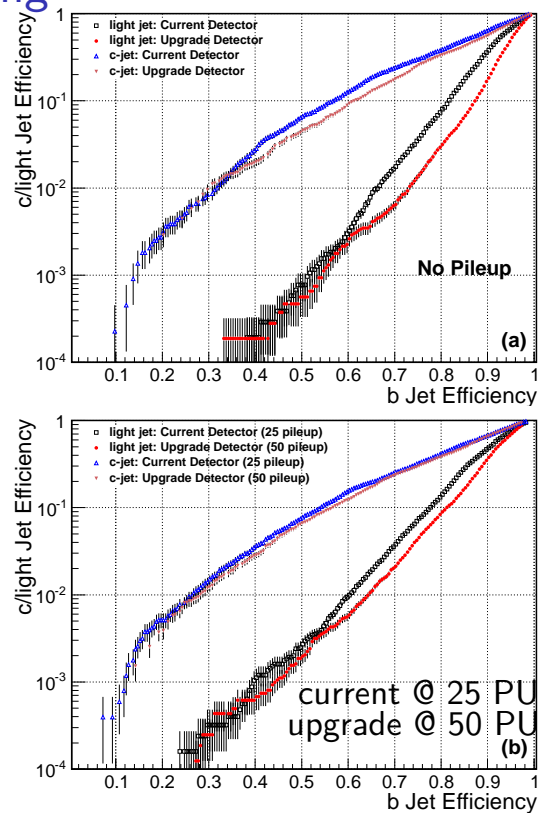


\section{Summary}

- Upgrade of CMS pixel detector motivated by excellent performance of LHC as well as accumulated radiation damage

- Additional detector layer, reduced material budged and improved readout will maintain or even improve performance of current pixel detector at pile-up of 50 or higher

- Upgrade can be performed with minimal impact on other detector components (i.e. no new cables or pipes required)

New technical design report for the CMS pixel upgrade available: http://cdsweb.cern.ch/record/1481838 